\title{
An unbalanced monocyte polarisation in peripheral blood and bone marrow of patients with type 2 diabetes has an impact on microangiopathy
}

\author{
G. P. Fadini • S. Vigili de Kreutzenberg • E. Boscaro • M. Albiero • \\ R. Cappellari • N. Kränkel • U. Landmesser • A. Toniolo • C. Bolego • \\ A. Cignarella - F. Seeger - S. Dimmeler • A. Zeiher - C. Agostini - A. Avogaro
}

Received: 2 January 2013 / Accepted: 5 April 2013 / Published online: 26 April 2013

(C) Springer-Verlag Berlin Heidelberg 2013

\begin{abstract}
Aim/hypothesis Monocytes/macrophages play important roles in adipose and vascular tissues and can be polarised as inflammatory M1 or anti-inflammatory M2. We sought to analyse monocyte polarisation status in type 2 diabetes, which is characterised by chronic inflammation.

Methods We enrolled 60 individuals without diabetes and 53 patients with type 2 diabetes. We quantified standard monocyte
\end{abstract}

Electronic supplementary material The online version of this article (doi:10.1007/s00125-013-2918-9) contains peer-reviewed but unedited supplementary material, which is available to authorised users.

G. P. Fadini $(\bowtie) \cdot S$. V. de Kreutzenberg $\cdot$ E. Boscaro $•$

C. Agostini $\cdot$ A. Avogaro

Division of Metabolic Diseases, Department of Medicine, University of Padova, Via Giustiniani, 2, 35100 Padova, Italy

e-mail: gianpaolo.fadini@unipd.it

G. P. Fadini • M. Albiero • R. Cappellari • C. Agostini •

A. Avogaro

Venetian Institute of Molecular Medicine (VIMM), Padova, Italy

N. Kränkel $\cdot$ U. Landmesser

Institute of Physiology, Cardiovascular Research, University of

Zurich, Zurich, Switzerland

N. Kränkel $\cdot$ U. Landmesser

Department of Cardiology, University Hospital Zurich, Zurich, Switzerland

\section{A. Toniolo $\cdot$ C. Bolego $\cdot$ A. Cignarella}

Department of Pharmaceutical Sciences, University of Padova,

Padova, Italy

F. Seeger $\cdot$ S. Dimmeler $\cdot$ A. Zeiher

Centre of Molecular Medicine, Institute for Cardiovascular

Regeneration, Frankfurt, Germany

F. Seeger $\cdot$ A. Zeiher

Department of Medicine III, Frankfurt University, Frankfurt, Germany subsets defined by cluster of differentiation (CD)14 and CD16. In addition, based on the phenotype of polarised macrophages in vitro, we characterised and quantified more definite M1 $\left(\mathrm{CD} 68^{+} \mathrm{CCR} 2^{+}\right)$and $\mathrm{M} 2\left(\mathrm{CX} 3 \mathrm{CR} 1^{+} \mathrm{CD} 206^{+} / \mathrm{CD} 163^{+}\right)$monocytes. We also analysed bone marrow (BM) samples and the effects of granulocyte-colony stimulating factor (G-CSF) stimulation in diabetic and control individuals.

Results We found no alterations in standard monocyte subsets (classical, intermediate and non-classical) when comparing groups. For validation of M1 and M2 phenotypes, we observed that $\mathrm{M} 2$ were enriched in non-classical monocytes and had lower TNF- $\alpha$ content, higher LDL scavenging and lower transendothelial migratory capacity than M1. Diabetic patients displayed an imbalanced M1/M2 ratio compared with the control group, attributable to a reduction in M2. The M1/M2 ratio was directly correlated with waist circumference and $\mathrm{HbA}_{1 \mathrm{c}}$ and, among diabetic patients, M2 reduction and $\mathrm{M} 1 / \mathrm{M} 2$ increase were associated with microangiopathy. A decrease in M2 was also found in the BM from diabetic patients, with a relative M2 excess compared with the bloodstream. BM stimulation with G-CSF mobilised M2 macrophages in diabetic but not in healthy individuals.

Conclusions/interpretation We show that type 2 diabetes markedly reduces anti-inflammatory M2 monocytes through a dysregulation in bone-marrow function. This defect may have a negative impact on microangiopathy.

Keywords Complications · Immune cells · Inflammation · Obesity

$\begin{array}{ll}\text { Abbreviations } \\ \text { APC } & \text { Allophycocyanin } \\ \text { BM } & \text { Bone marrow } \\ \text { CCR2 } & \text { Chemokine-C receptor-2 } \\ \text { CD } & \text { Cluster of differentiation }\end{array}$




$\begin{array}{ll}\text { CX3CR1 } & \text { Chemokine-X3C receptor-1 } \\ \text { DiI-AcLDL } & \text { DiI-labelled acetylated LDL } \\ \text { eGFR } & \text { Estimated glomerular filtration rate } \\ \text { G-CSF } & \text { Granulocyte-colony stimulating factor } \\ \text { HAEC } & \text { Human aortic endothelial cells } \\ \text { LPS } & \text { Lipopolysaccharide } \\ \text { MAb } & \text { Monoclonal antibody } \\ \text { MCP-1 } & \text { Monocyte chemotactic protein-1 } \\ \text { PB } & \text { Peripheral blood } \\ \text { PBMC } & \text { Peripheral blood mononuclear cells } \\ \text { PE } & \text { Phycoerythrin }\end{array}$

\section{Introduction}

Metabolic diseases are characterised by chronic systemic inflammation but the mechanisms involved are unclear [1, 2]. In response to dietary challenges, adipocytes and endothelial cells activate classical inflammatory pathways, which impair the metabolic action of insulin, paving the way to diabetes [3]. In turn, hyperglycaemia and hyperinsulinaemia elicit multiple pro-inflammatory responses [4-6]. This is reflected by mild elevation of inflammatory markers in patients with diabetes or the metabolic syndrome [7]. Inflammation also promotes development and progression of diabetic complications, including atherogenesis, nephropathy, retinopathy and neuropathy [8-11].

One pivotal aspect of innate immunity and chronic inflammation is the polarisation of monocyte/macrophages, which are endowed with remarkable plasticity [12]. Tissue macrophages exist in two major states - classically activated inflammatory M1 and alternatively activated anti-inflammatory M2 [13]. Roughly, M1 macrophages express the cluster of differentiation (CD)68 macrophage marker and the chemokine-C receptor-2 (CCR2), while M2 macrophages typically express the macrophage marker chemokine-X3C receptor-1 (CX3CR1) and scavenger receptors CD206 and CD163 [14]. It has been shown that M1 and M2 macrophages exert different actions in obesity and atherosclerosis $[14,15]$. For instance, adipose tissue macrophages display a prevalent M1 phenotype, which sustains inflammation and can be shifted toward M2 after weight loss [16]. In atherosclerosis, M1 macrophages are prone to become foam cells, while M2 cells exert scavenger activity and suppress inflammation [17]. To a similar extent, circulating monocytes are believed to reflect different inflammatory states. Based on CD14 and CD16 expression, the standard monocyte nomenclature distinguishes classical $\left(\mathrm{CD} 14^{++} \mathrm{CD} 16\right)$, intermediate $\left(\mathrm{CD} 14^{++} \mathrm{CD} 16^{+}\right)$and non-classical $\left(\mathrm{CD} 14^{+} \mathrm{CD} 16^{+}\right)$ cells, with distinctive gene expression profiles $[18,19]$.

To date, the polarisation status of circulating monocytes in type 2 diabetes is poorly characterised and has been mainly derived from gene expression analyses $[20,21]$. In this study, we aimed to determine the pro-inflammatory vs anti-inflammatory monocyte polarisation balance in patients with type 2 diabetes compared with control individuals, by identifying, characterising and quantifying novel discrete cell populations.

\section{Methods}

Patients

The protocol was approved by the local ethical committee and conducted in accordance with the Declaration of Helsinki as revised in 2000. Diabetic and control individuals were recruited at the Division of Metabolic Diseases of the University Hospital of Padova. All consecutive patients were deemed eligible, pending provision of informed consent and meeting inclusion/exclusion criteria. Type 2 diabetes was defined according to ADA criteria [22]. Control individuals had normal fasting plasma glucose and $\mathrm{HbA}_{1 \mathrm{c}}$ (International Federation of Clinical Chemistry [IFCC]-aligned HPLC method) $<6.0 \%(<42 \mathrm{mmol} / \mathrm{mol})$. Individuals aged 25 80 years could be included. Exclusion criteria were: acute disease or infection, immunosuppression/organ transplantation, chronic inflammatory diseases, recent surgery, trauma, cardiovascular events and pregnancy/lactation. For all patients, we recorded the following data: age, sex, BMI, waist circumference, blood pressure, history of hypertension, smoking, lipid profile, fasting plasma glucose, $\mathrm{HbA}_{1 \mathrm{c}}$ and creatinine levels. Diabetic retinopathy was diagnosed by digital funduscopy. Diabetic nephropathy was defined as a urinary albumin/creatinine ratio $>3.4 \mathrm{mg} / \mathrm{mmol}$ or an estimated glomerular filtration rate (eGFR) $<60 \mathrm{ml} \mathrm{min}^{-1} 1.73 \mathrm{~m}^{-2}$. Coronary artery disease was defined as a history of myocardial infarction or angina, confirmed by coronary angiography or a myocardial stress test. Peripheral arterial disease was defined as a history of claudication or rest pain with evidence of leg artery stenosis upon invasive or non-invasive examination. Cerebrovascular disease was defined as a history of stroke or evidence of $>30 \%$ carotid stenosis at ultrasound examination. Atherosclerosis/macroangiopathy was defined as the presence of either coronary, peripheral or cerebrovascular disease. We also collected data on medications.

\section{Bone marrow samples}

Patients with heart disease, with $(n=5)$ and without $(n=5)$ type 2 diabetes, recruited at the Johann Wolfgang Goethe University Hospitals for cell therapy, were included. The ethics review board of Goethe University approved the protocols (NCT00962364 and NCT00284713). After giving informed consent, before cell therapy, patients were subjected to aspiration of bone marrow (BM) and about $1 \mathrm{ml}$ of the $\mathrm{BM}$ aspirate was collected for M1/M2 quantification. 


\section{BM stimulation}

In a prospective clinical trial (NCT01102699), we enrolled 13 individuals with type 2 diabetes and 14 non-diabetic individuals, who underwent BM stimulation with a single subcutaneous injection of $5 \mu \mathrm{g} / \mathrm{kg}$ filgrastim (Granulokine; Amgen, Thousand Oaks, CA, USA) [23]. Before and $24 \mathrm{~h}$ after injection, blood samples were drawn for determination of $\mathrm{M} 1 / \mathrm{M} 2$.

Identification and characterisation of monocyte subsets

Identification of monocyte subsets was performed using multiparameter flow cytometry. For analysis of classical, intermediate and non-classical monocytes, cells were stained with an FITC or phycoerythrin (PE) anti-CD14 monoclonal antibody (mAb) (BD Pharmingen, Franklin Lakes, NJ, USA) and an FITC- or PE-Cy5 anti-CD16 mAb (Beckman Coulter, Pasadena, CA, USA). The analysis was performed according to standardised gating strategy [18]. For more definite monocyte subsets, we stained with FITC anti-CD68 mAb (Dako, Milan, Italy) and PE or AlexaFluor-647 anti-CCR2 mAb (R\&D Systems, Minneapolis, MN, USA) for identification of M1 cells and with FITC anti-CX3CR1 (Biolegend, San Diego, CA, USA), PE anti-CD163 (BD) and allophycocyanin (APC) anti-CD206 (BD) mAbs for M2. M1 cells were defined as $\mathrm{CD} 68^{+} \mathrm{CCR} 2^{+}$cells and $\mathrm{M} 2$ cells were defined as $\mathrm{CX} 3 \mathrm{CR} 1^{+} \mathrm{CD} 163^{+} / \mathrm{CD} 206^{+}$. In preliminary experiments, we found a strong correlation between CD163 and CD206 staining on $\mathrm{CX} 3 \mathrm{CR} 1^{+}$cells, suggesting that expression of these scavenger receptors on monocytes convey similar information. Thus, M2 were defined as $\mathrm{CX} 3 \mathrm{CR} 1^{+}$cells that express either CD163 or CD206. The relative frequency of these monocyte subsets were expressed as the percentage of the total monocyte gate. Reproducibility of M1 and M2, assessed as $\mathrm{CV}$ in a test-retest sample of ten individuals was $7.8 \%$ and $8.9 \%$, respectively.

In separate experiments performed with peripheral blood of healthy individuals, M1 and M2 cells were stained with CD14 and CD16. Gated CD $68^{+} \mathrm{CCR}^{+}$(M1) and $\mathrm{CX} \mathrm{CR} 1^{+} \mathrm{CD} 206^{+}$(M2) cells were tested against standard monocyte subsets, allowing the positioning of M1 and M2 cells within the traditional CD14/CD16 monocyte subsets.

For the study of phagocytosis of modified LDL particles, $\mathrm{M} 1\left(\mathrm{CD} 68^{+} \mathrm{CCR} 2^{+}\right)$and $\mathrm{M} 2\left(\mathrm{CX} 3 \mathrm{CR} 1^{+} \mathrm{CD} 206^{+}\right)$cells were incubated with $10 \mu \mathrm{g} / \mathrm{ml}$ DiI-labelled acetylated LDL (DiIAcLDL; Molecular Probes, Eugene, OR, USA) for $2 \mathrm{~h}$ at $37^{\circ} \mathrm{C}$. The percentage of cells positive for DiI-AcLDL in the M1 or M2 gate was analysed.

We also quantified the expression of pro-inflammatory (TNF- $\alpha$ ) and anti-inflammatory (IL-10) cytokines on M1 and M2 cells. To this aim, cells were stained with M1 or M2 markers, permeabilised and stained with APC-conjugated anti-human TNF- $\alpha$ or IL-10 (BD) after $2 \mathrm{~h}$ incubation with $2 \mu \mathrm{mol} / 1 \mathrm{monensin}$. The percentage of M1 $\left(\mathrm{CD} 68^{+} \mathrm{CCR} 2^{+}\right)$ or M2 $\left(\mathrm{CX} 3 \mathrm{CR} 1^{+} \mathrm{CD} 163^{+}\right)$cells expressing TNF- $\alpha$ or IL-10 was calculated.

M1 $\left(\mathrm{CD} 8^{+} \mathrm{CCR} 2^{+}\right)$and $\mathrm{M} 2\left(\mathrm{CX} 3 \mathrm{CR} 1^{+} \mathrm{CD} 163^{+}\right)$cells were also analysed for expression of granulocyte-colony stimulating factor (G-CSF) receptor CD114 using an APCconjugated anti-CD114 mAb (BD).

Primary culture of polarised macrophages

Venous blood was obtained from healthy donors and separated using a Ficoll-Paque solution. Mononuclear cells were collected, washed with PBS containing EDTA (5 mmol/l) and resuspended at $2 \times 10^{6}-3 \times 10^{6}$ cells $/ \mathrm{ml}$ in RPMI-1640 supplemented with glutamine, penicillin-streptomycin and $15 \%$ FCS. Monocytes were separated from lymphocytes by adherence to $100-\mathrm{mm}$ plastic dishes for $2 \mathrm{~h}$. Adherent monocytes were cultured in fresh medium for 7 days at $37^{\circ} \mathrm{C}$ to allow spontaneous differentiation into macrophages. Then, resting cells were polarised into M1 or M2 macrophages by incubation for $48 \mathrm{~h}$ with lipopolysaccharide (LPS; $1 \mu \mathrm{g} / \mathrm{ml}$ ) and IFN- $\gamma(10 \mathrm{ng} / \mathrm{ml})$ or IL-4 $(20 \mathrm{ng} / \mathrm{ml})$ and IL-13 $(5 \mathrm{ng} / \mathrm{ml})$, respectively. In separate experiments, M1 and M2 stimuli were added at the beginning of the culture, for 7 days.

Ex vivo stimulation of monocytes

Peripheral blood mononuclear cells (PBMCs) were obtained as described above. Cells were incubated for $3 \mathrm{~h}$ at $37^{\circ} \mathrm{C}$, then non-adherent cells were discarded and the remaining adherent fraction (monocytes) was incubated with $20 \mathrm{ng} / \mathrm{ml}$ of recombinant G-CSF for $24 \mathrm{~h}$. Control cells were incubated with an equal volume of PBS. After treatment, cells were washed twice with PBS to remove non-adherent cells and detached for analysis.

Transendothelial migration

To test the ability of M1 and M2 cells to undergo transendothelial migration, we used a modified transwell migration assay [24]. Freshly isolated human PBMCs were left to adhere to, or transmigrate through, a monolayer of red PKH26-labelled (Sigma Aldrich, St Louis, MO, USA) human aortic endothelial cells grown to confluence on the filter membrane of sterile $3 \mu \mathrm{m}$-pore, 12-well format transwell migration inserts (BD). After $6 \mathrm{~h}$, we collected transmigrating PBMCs from the lower compartment (migrated fraction) and adherent cell population by sequential washing/detachment. The two cell fractions (adherent and migrated) were stained with $\mathrm{M} 1\left(\mathrm{CD} 68^{+} \mathrm{CCR} 2^{+}\right)$and $\mathrm{M} 2$ $\left(\mathrm{CX} 3 \mathrm{CR} 1^{+} \mathrm{CD} 206^{+}\right)$markers to look for enrichment of M1 and M2 cells in the migrated vs adherent fraction. 
Endothelial cells were separated from PBMCs during the analysis by a multiparametric gating strategy.

Statistical analyses

Data are expressed as mean $\pm \mathrm{SE}$ or as percentage. Normality of the variables of interest were checked with the Kolmogorov-Smirnov test. Non-normal variables were logtransformed for analyses. Comparisons between two groups were performed using Student's $t$ test for normal variables and with Mann-Whitney's $U$ test for non-normal variables. Percentages were compared using the $\chi^{2}$ test. Multiple linear regression analyses were performed by entering cell levels as the dependent variable and clinical characteristics as independent variables in a single block. Explanatory variables were selected as those that were significantly different upon univariate group comparison in Table 1 plus sex, but medications were not included to avoid overfitting.
Statistical significance was accepted at $p<0.05$; SPSS (IBM Corporation, Armonk, NY, USA) version 18 was used.

\section{Results}

Standard CD14/CD16 monocyte subsets in diabetic patients and control individuals

In 60 control individuals with normal glucose metabolism and 53 patients with type 2 diabetes, we determined the percentages of classical $\left(\mathrm{CD} 14^{++} \mathrm{CD} 16^{-}\right)$, intermediate $\left(\mathrm{CD} 14^{++} \mathrm{CD} 16^{+}\right)$and non-classical $\left(\mathrm{CD} 14^{+} \mathrm{CD} 16^{+}\right)$monocytes (Fig. 1a, b), as well as of $\mathrm{M} 1\left(\mathrm{CD} 68^{+} \mathrm{CCR} 2^{+}\right)$and $\mathrm{M} 2$ $\left(\mathrm{CX} 3 \mathrm{CR} 1^{+} \mathrm{CD} 163^{+} / \mathrm{CD} 206^{+}\right)$cells. Clinical characteristics are reported in Table 1. In control individuals, classical, intermediate and non-classical monocytes accounted for $32.4 \pm 4.4 \%, 45.4 \pm 4.3 \%$ and $7.8 \pm 0.6 \%$, respectively. We
Table 1 Characteristics of the study population
Data are presented as means \pm SE unless indicated otherwise

${ }^{*} p<0.05$ vs control

ACEi, angiotensin-converting enzyme inhibitor; ARB, angiotensin receptor blocker; CVD, cardiovascular disease

\begin{tabular}{|c|c|c|}
\hline Variable & Control & Type 2 diabetes \\
\hline$n$ & 60 & 53 \\
\hline Age, years & $57.6 \pm 1.3$ & $61.6 \pm 1.3 *$ \\
\hline Sex male, $n(\%)$ & $31(51.7)$ & $38(69.8)$ \\
\hline BMI, $\mathrm{kg} / \mathrm{m}^{2}$ & $26.1 \pm 1.3$ & $28.5 \pm 0.8^{*}$ \\
\hline Fasting plasma glucose, $\mathrm{mmol} / \mathrm{l}$ & $5.08 \pm 0.06$ & $8.67 \pm 0.32 *$ \\
\hline \multicolumn{3}{|l|}{$\mathrm{HbA}_{1 \mathrm{c}}$} \\
\hline$\%$ & $5.5 \pm 0.1$ & $7.7 \pm 1.2$ \\
\hline $\mathrm{mmol} / \mathrm{mol}$ & $36.6 \pm 0.7$ & $60.7 \pm 9.5^{*}$ \\
\hline Hypertension, $n(\%)$ & $20(33.9)$ & $41(77.3)^{*}$ \\
\hline Systolic blood pressure, $\mathrm{mmHg}$ & $134.0 \pm 2.5$ & $139.2 \pm 2.6$ \\
\hline Diastolic blood pressure, $\mathrm{mmHg}$ & $84.8 \pm 1.4$ & $81.3 \pm 1.3$ \\
\hline Total cholesterol, mmol/1 & $5.21 \pm 0.16$ & $4.41 \pm 0.13^{*}$ \\
\hline HDL-cholesterol, mmol/1 & $1.52 \pm 0.06$ & $1.26 \pm 0.06^{*}$ \\
\hline LDL-cholesterol, mmol/1 & $3.04 \pm 0.13$ & $2.49 \pm 0.1^{*}$ \\
\hline Triacylglycerols, mmol/1 & $1.42 \pm 0.13$ & $1.44 \pm 0.1$ \\
\hline Urinary albumin/creatinine ratio, $\mathrm{mg} / \mathrm{mmol}$ & $0.34 \pm 0.1$ & $17.1 \pm 10.3^{*}$ \\
\hline Serum creatinine, $\mu \mathrm{mol} / 1$ & $81.8 \pm 3.6$ & $90.9 \pm 4.8$ \\
\hline eGFR $<60 \mathrm{ml} \mathrm{min}{ }^{-1} 1.73 \mathrm{~m}^{-2}, n(\%)$ & $0(0.0)$ & $6(11.3)^{*}$ \\
\hline Retinopathy, $n(\%)$ & $0(0.0)$ & $11(20.7)^{*}$ \\
\hline Coronary artery disease, $n(\%)$ & $2(3.3)$ & $12(22.6)^{*}$ \\
\hline Atherosclerotic CVD, $n(\%)$ & $5(8.3)$ & $27(50.9)^{*}$ \\
\hline \multicolumn{3}{|l|}{ Medication } \\
\hline Insulin, $n(\%)$ & $0(0.0)$ & $35(66.0)^{*}$ \\
\hline Metformin, $n(\%)$ & $0(0.0)$ & $36(67.9)^{*}$ \\
\hline Secretagogues, $n(\%)$ & $0(0.0)$ & $23(43.4)^{*}$ \\
\hline Incretin drugs, $n(\%)$ & $0(0.0)$ & $4(7.5)^{*}$ \\
\hline ACEi/ARBs, $n(\%)$ & $17(28.3)$ & $40(75.5)^{*}$ \\
\hline Other hypotensives, $n(\%)$ & $10(16.7)$ & $30(56.6)^{*}$ \\
\hline Anti-aggregants, $n(\%)$ & $11(18.3)$ & $30(56.6)^{*}$ \\
\hline Statins, $n(\%)$ & $32(53.3)$ & $33(62.2)$ \\
\hline
\end{tabular}


a

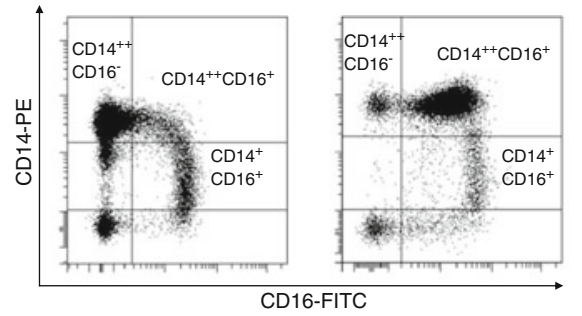

C

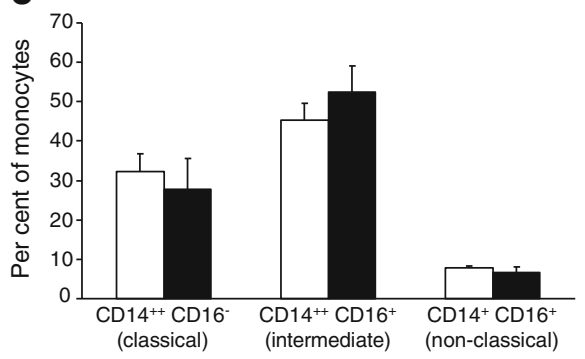

d

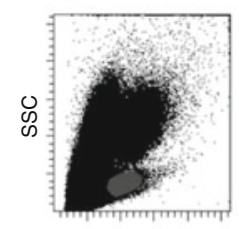

FSC

e

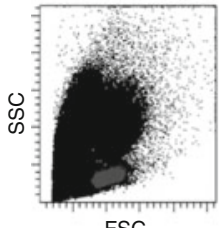

FSC

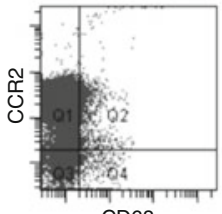

CD68

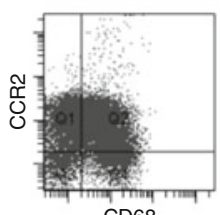

CD68
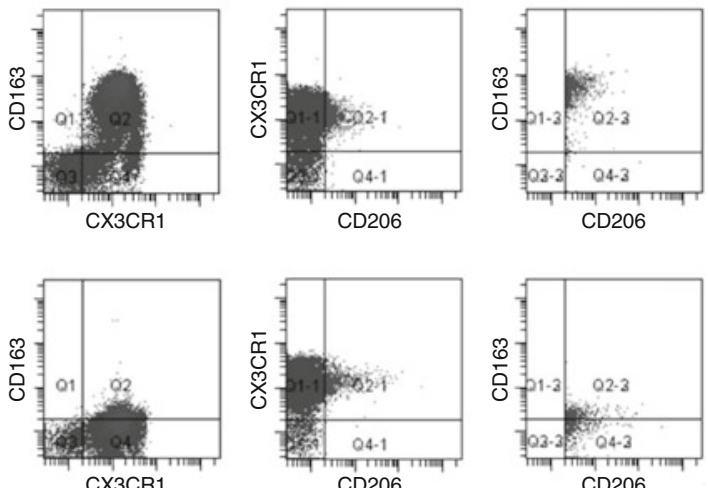

f
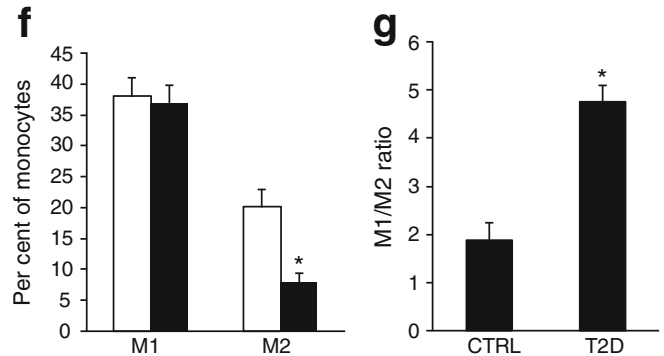

h

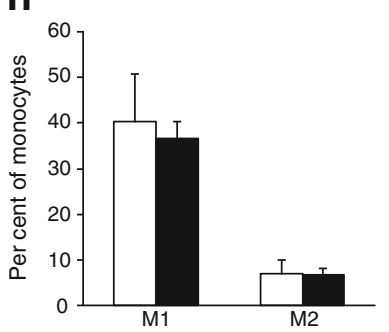

i

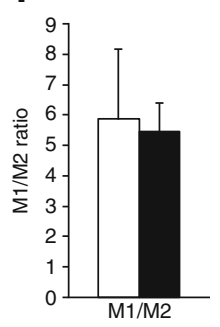

Fig. 1 Analysis of traditional and novel monocyte subsets. (a-c) In individuals with and without type 2 diabetes, we quantified the traditional monocyte subsets by CD14 and CD16 staining. Representative scatter plots of a patient with predominance of classical $\mathrm{CD} 14^{++} \mathrm{CD} 16^{-}$monocytes (a) and of a patient with prevalence of intermediate $\mathrm{CD} 14^{++} \mathrm{CD} 16^{+}$ monocytes (b). Quantification of these phenotypes did not identify any significant difference between controls (white bars) and type 2 diabetes (black bars) (c). (d, e) The gating strategy was used to identify and quantify circulating $\mathrm{CD} 68^{+} \mathrm{CCR}^{+}(\mathrm{M} 1)$ and $\mathrm{CX} 3 \mathrm{CR} 1^{+} \mathrm{CD} 206^{+} / \mathrm{CD} 163^{+}$(M2)

found no significant differences in these monocyte subsets when comparing patients with type 2 diabetes and control individuals (Fig. 1c).

Functional characteristics of circulating M1 and M2 monocytes

Based on the negative results of the analysis of standard CD14/CD16 monocyte subsets, we moved to analyse cells expressing more definite M1 (CD68 and CCR2) or M2 (CX3CR1, CD163 and CD206) markers (Fig. 1d, e). We compared these circulating cells with monocyte-macrophage cells in an individual without (d) and with (e) type 2 diabetes. (f) We quantified M1 $\left(\mathrm{CD}^{+} 8^{+} \mathrm{CCR} 2^{+}\right)$and $\mathrm{M} 2\left(\mathrm{CX} 3 \mathrm{CR} 1^{+} \mathrm{CD} 206^{+} / \mathrm{CD} 163^{+}\right)$ cells in control individuals (white bars) and patients with type 2 diabetes (black bars). ${ }^{*} p<0.05$ vs control. (g) The M1/M2 ratio was calculated to represent the monocyte polarisation balance in non-diabetic individuals (CTRL) and patients with type 2 diabetes (T2D). (h, i) Comparison between drug-naive diabetic patients ( $n=10$, white bars) and other diabetic patients included in the study ( $n=43$, black bars). ${ }^{*} p<0.05$ vs CTRL

phenotypes and performed a functional characterisation for validation.

Analogy to in vitro polarised macrophages We first determined whether the M1 and M2 markers used here to define monocytes are expressed by cultured macrophages polarised in vitro using standard protocols. M1 cells obtained after incubating monocytes of healthy donors with LPS and IFN- $\gamma$ showed a $3.6 \pm 1.2$-fold increase in $\mathrm{CD} 68$, a $74.9 \pm$ $8.5 \%$ reduction in $\mathrm{CD} 206$ and an $87.4 \pm 5.8 \%$ reduction in CD163 expression compared with unstimulated cells, while CCR2 did not significantly change. In M2 cells obtained by 
stimulation with IL-4+IL-13, CD206 and CD163 expres-

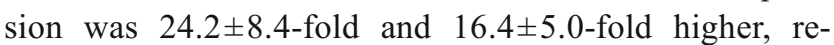
spectively, than in M1 cells. M2 markers were increased after a 7-day stimulation compared with unstimulated cells (electronic supplementary material [ESM] Fig. 1a). These data indicate that the M1 and M2 markers used to define monocyte subsets resemble macrophage phenotypes in vitro, with the exception of chemokine receptors CCR2 and CX3CR1.

Comparison of monocyte-macrophage gene expression between M1 and M2 cells and in vitro polarised macrophages was also performed in silico using public resources (GEO accession number GSE5099 [25]). While the chemokine receptor genes CCR2 and $C X 3 C R 1$ are rapidly downregulated during monocytemacrophage differentiation, $C D 206$ and CD16 (also known as $F C G R 3 A / B$ ) are overexpressed in M2 compared with M1 macrophages and can be considered genes distinctive of the M2 phenotype both in the blood and in vitro (ESM Fig. 2a).

Comparison with CD14/CD16 monocytes To further clarify the identity of M1 and M2 monocytes defined by the selected antigenic profiles, we stained $\mathrm{CD} 68^{+} \mathrm{CCR} 2^{+}$(M1) and $\mathrm{CX} \mathrm{CR} 1^{+} \mathrm{CD} 206^{+}$(M2) cells with $\mathrm{CD} 14$ and CD16, which allows a standardised definition of monocyte subsets. We found that the percentage of cells belonging to the classical $\mathrm{CD} 14^{++} \mathrm{CD} 16^{-}$monocytic fraction was twofold higher for M1 compared with M2 $(22.8 \pm 2.3 \%$ vs $11.3 \pm 4.2 \%$; $p=$ $0.05)$, while the percentage of cells belonging to nonclassical $\mathrm{CD} 14^{+} \mathrm{CD} 16^{+}$monocytes was fourfold higher for M2 compared with M1 $(8.4 \pm 1.8 \%$ vs $2.0 \pm 0.8 \% ; p=0.03)$ (ESM Fig. 1b). These data indicate that M1 and M2 cells have different monocytic features, and support the definition of $\mathrm{CD} 68^{+} \mathrm{CCR} 2^{+}$cells as classical inflammatory monocytes and $\mathrm{CXC} 3 \mathrm{CR} 1^{+} \mathrm{CD} 206^{+}$cells as anti-inflammatory cells [26]. The analysis of gene expression from public resources (GEO accession number GSE25913 [19]) confirms that CX3CR1 is about 2.2-fold overexpressed in $\mathrm{CD} 14^{++} \mathrm{CD} 16^{+}$ compared with $\mathrm{CD} 14^{++} \mathrm{CD} 16^{-}$monocytes, while $C D 163$ appears to be expressed at a higher level in $\mathrm{CD} 16^{-}$compared with $\mathrm{CD}_{16}{ }^{+}$cells (ESM Fig. 2b). These data suggest that the analysis of surface protein expression by flow cytometry provides complementary information over and beyond gene expression.

Cytokine expression We analysed the expression of TNF- $\alpha$ and IL-10 by intracellular flow cytometry. We found that $8.5 \pm$ $2.9 \%$ of $\mathrm{M} 1\left(\mathrm{CD} 8^{+} \mathrm{CCR} 2^{+}\right)$and $2.0 \pm 0.7 \%$ of $\mathrm{M} 2$ $\left(\mathrm{CX} 3 \mathrm{CR} 1^{+} \mathrm{CD} 163^{+}\right)$cells were brightly positive for TNF- $\alpha(p<0.05)$, while the expression of IL-10 was not significantly different (M1 $7.6 \pm 3.0 \%$ vs M2 $5.6 \pm 1.1 \%$; $p=0.72)$ (ESM Fig. 1c).
Uptake of modified LDL After staining peripheral blood cells with DiI-AcLDL, we found that about $5 \%$ of circulating monocytes took up acetylated LDL while lymphocytes were negative for DiI-AcLDL uptake, indicating that a fraction of monocytes had phagocytic activity. We found that $\mathrm{CX} 3 \mathrm{CR} 1^{+} \mathrm{CD} 206^{+} \mathrm{M} 2$ cells showed a $40.8 \pm 14.3 \%$ increased LDL uptake compared with $\mathrm{CD} 68^{+} \mathrm{CCR} 2^{+} \mathrm{M} 1$ cells $(p=0.02)$ (ESM Fig. 1d). This is consistent with the notion that alternatively activated macrophages (M2) have a distinctive phagocytic activity [28], possibly related to the higher expression of scavenger receptors.

Transendothelial migration Spontaneous migration across an endothelial layer, as well as monocyte chemotactic protein-1 (MCP-1) or fractalkine directed migration, was studied and the ratios between the percentages of cells staining for M1 or M2 markers in the migrated vs the adherent compartment were compared. This ratio was significantly higher for M1 than for M2 cells (ESM Fig. 1e), indicating that M1 cells have a higher spontaneous and chemokine-induced transendothelial migratory capacity than M2. The amount of cells migrated toward chemokines was underestimated owing to chemokine receptor (CCR2 and CX3CR1) internalisation during ligand stimulation. Therefore, that M1 migrated more than M2, independently of the agonist used, points to the intrinsically higher migratory capacity of M1 than M2.

\section{Circulating M1/M2 polarisation balance in diabetes}

The validation analysis indicates that circulating M1 and M2 cells share characteristics with in vitro polarised macrophages and have different functions. Therefore, we quantified these monocyte phenotypes in patients with type 2 diabetes vs healthy controls.

In healthy controls, $38.0 \pm 3.0 \%$ of circulating monocytes had an M1 phenotype and $20.2 \pm 2.3 \%$ satisfied M2 criteria. Thus, the normal M1/M2 polarisation balance (M1/M2 ratio) was 1.9 . The remaining $\sim 40 \%$ of monocytes did not satisfy either M1 or M2 criteria, indicating they were less differentiated. Patients with type 2 diabetes showed a striking reduction in $\mathrm{M} 2\left(7.7 \pm 1.6 \% ; p=4 \times 10^{-5}\right)$ with no change in M1 $(36.9 \pm 2.9 \% ; p=0.79$; Fig. 1f). As a result, the M1/M2 ratio was markedly increased in type 2 diabetes $(4.8 \pm 0.8 ; p<0.001)$ (Fig. 1g). Diabetic patients were older and had a worse risk profile than controls, but the decrease in M2 remained significantly associated with diabetes upon correction for confounders (ESM Table 1). We also analysed separately ten drug-naive diabetic patients with a shorter disease duration $(3.2 \pm 1.4$ vs $12.3 \pm 3.8$ years; $p<0.01$ ) and found that $\mathrm{M} 1$ and $\mathrm{M} 2$ levels, as well as the M1/M2 ratio, were similar to the rest of the diabetic population (Fig. 1h, i). 
These data indicate that while the traditional monocyte nomenclature is poorly informative, type 2 diabetes affects the pattern of M1/M2 monocyte polarisation, which is characterised by M2 deficiency.

Relationships with clinical characteristics and complications

In the whole population, we found that the $\mathrm{M} 1 / \mathrm{M} 2$ ratio was correlated with waist circumference $(r=0.22 ; p=0.018)$ and $\mathrm{HbA}_{1 \mathrm{c}}(r=0.26 ; p=0.005)$. We then looked for associations between the type and severity of complications and alterations in monocyte subtypes. While M1 cells showed no consistent trend in relation to complications (Fig. 2a), we found a trend toward decreased M2 in patients with nephropathy or retinopathy (Fig. 2b). The M1/M2 ratio was significantly reduced in patients with nephropathy and a trend was detected for retinopathy (Fig. 2c). When patients were divided according to the presence of microangiopathy or macroangiopathy, or both, M2 levels and the M1/M2 appeared to be related to microangiopathy (Fig. 2d-f).

\section{Monocyte phenotypes in diabetic BM}

We then examined the percentage of M1 and M2 cells in $\mathrm{BM}$ aspirates of five individuals with type 2 diabetes and five control individuals (ESM Table 2). In control individuals, M1/M2 polarisation in the BM was inverted compared with peripheral blood, with a predominance of M2 (29.3 \pm

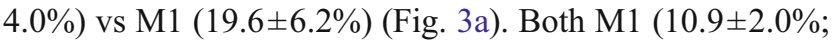
$p=0.23)$ and $\mathrm{M} 2(16.4 \pm 2.0 \% ; p=0.029)$ were reduced in the diabetic BM but only the M2 reduction was statistically significant. The BM M1/M2 polarisation balance was identical in diabetic and control individuals (Fig. 3b).

Despite $\mathrm{M} 2$ reduction in the diabetic $\mathrm{BM}$, the percentage of M2 was much higher than in peripheral blood (PB). As monocytes are mobilised from the $\mathrm{BM}$ to the $\mathrm{PB}$, we determined the $\mathrm{BM} / \mathrm{PB}$ ratio in individuals with and without type 2 diabetes, as an indicator of the gradient between these two compartments. Compared with controls, diabetic patients showed a relative excess of M2 cells in the BM (Fig. 3c).

\section{G-CSF mobilises BM M2 in diabetes}

Given the different M1 and M2 levels in the BM of patients with type 2 diabetes compared with controls, we quantified circulating M1 and M2 cells in diabetic patients and controls undergoing BM stimulation with G-CSF in the clinical trial NCT01102699 (ESM Table 3). Twenty-four hours after GCSF injection, the M1/M2 remained stable in controls (Fig. 3d) and dropped in diabetic patients (Fig. 3e). This was attributable to an increase in M2 after G-CSF only in diabetic patients. These data indicate that G-CSF reverses the M1/M2 alterations in diabetes and indirectly confirm the relative excess of M2 in the BM. To understand why G-CSF mobilises M2 cells preferentially, we analysed the surface expression of the G-CSF receptor CD114 and found that $85.2 \pm 2.2 \%$ of $\mathrm{M} 1\left(\mathrm{CD} 68^{+} \mathrm{CCR} 2^{+}\right)$and $83.6 \pm 3.1 \%$ of $\mathrm{M} 2$ $\left(\mathrm{CX} 3 \mathrm{CR} 1^{+} \mathrm{CD} 163^{+}\right)$expressed CD114 $(p=0.39)(\mathrm{ESM}$ Fig. 3a). This rules out the possibility that different G-CSF receptor expression on $\mathrm{M} 1$ and $\mathrm{M} 2$ cells accounts for the differential effect of G-CSF on M2 vs M1 mobilisation. To rule out that the effect of G-CSF on the M1/M2 balance was
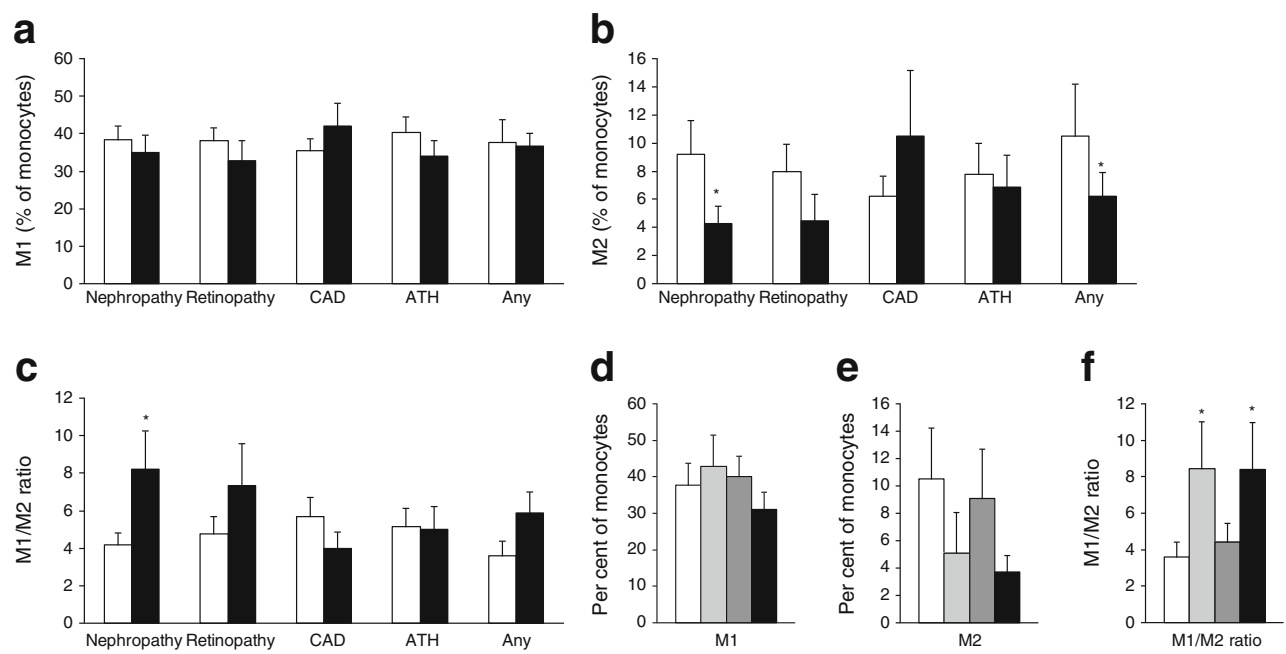

Fig. 2 Levels of M1 and M2 cells and M1/M2 ratio in relation to diabetic complications. (a-c) Percentage of M1 (a) and M2 monocytes (b) and the M1/M2 polarisation ratio (c) in patients with type 2 diabetes divided according to the absence (white bars) or presence (black bars) of diabetic complications. ${ }^{*} p<0.05$ vs absence of complication. (d-f) Percentage of M1 (d) and M2

monocytes (e) and the M1/M2 polarisation ratio (f) in patients with type 2 diabetes without complications (white bars), and in those with macroangiopathy alone (light-grey bars), with microangiopathy alone (dark-grey bars) and with both microand macroangiopathy (black bars). $* p<0.05$ vs absence of complication. ATH, atherosclerosis; CAD, coronary artery disease 

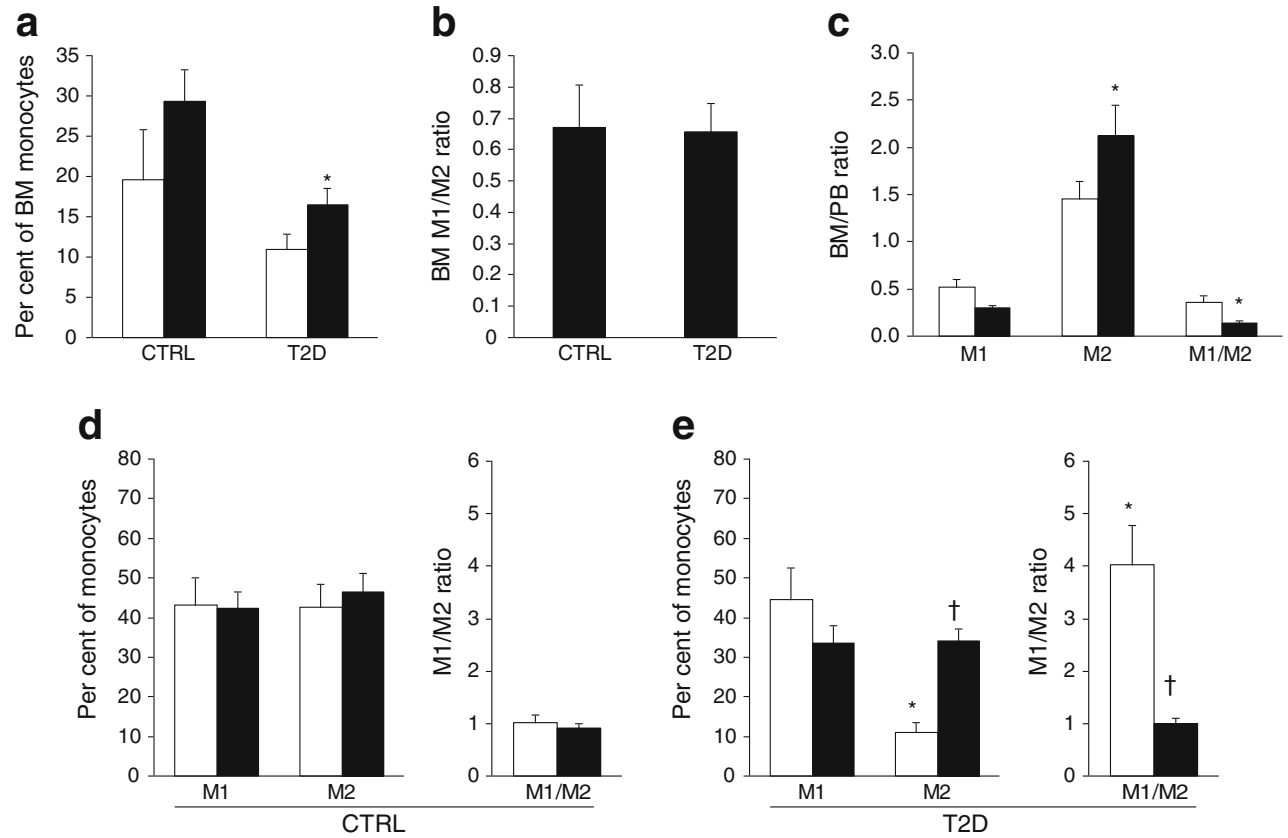

Fig. 3 M1 and M2 cells in BM. (a) M1 (white bars) and M2 (black bars) cells were quantified in BM samples of five control individuals (CTRL) and five patients with type 2 diabetes (T2D). ${ }^{*} p<0.05$ vs CTRL. (b) The M1/M2 ratio in the BM was calculated for controls and patients with type 2 diabetes. (c) The BM/PB ratio, as an index of the gradient between these two compartments, was calculated for M1,

due to direct polarisation rather than mobilisation, we treated ex vivo monocytes from individuals with or without type 2 diabetes for $24 \mathrm{~h}$ with the peak concentrations of G-CSF that are achieved in vivo after injection of $5 \mu \mathrm{g} / \mathrm{kg}$ (i.e. $20 \mathrm{ng} / \mathrm{ml}$ ), and found no significant effect of G-CSF on the M1/M2 ratio (ESM Fig. 3b). These results are consistent with the notion that G-CSF does not directly affect function of the cells that are mobilised [27].

\section{Discussion}

By studying novel phenotypes of monocyte polarisation, we show that type 2 diabetes is associated with a marked reduction of the anti-inflammatory M2 phenotype. Traditionally, monocyte subsets are defined by the expression of CD14 (LPS receptor) and CD16 (Fc $\gamma$ RIII) [18]. Previous studies reported changes in CD14/CD16expressing monocytes in relation to obesity and weight loss [28] but the effect of diabetes is controversial [29, 30]. With this standard nomenclature, we found no significant alteration in classical $\mathrm{CD} 14^{++} \mathrm{CD} 16^{-}$, intermediate $\mathrm{CD} 14^{++} \mathrm{CD} 16^{+}$and non-classical $\mathrm{CD} 14^{+} \mathrm{CD} 16^{+}$ monocytes in patients with type 2 diabetes vs controls. Specific characteristics of the cohorts under investigation may account for partial inconsistency with the

$\mathrm{M} 2$ and M1/M2 ratio in controls (white bars) and patients with type 2 diabetes (black bars). ${ }^{*} p<0.05$ vs controls. (d, e) M1 and M2 cell levels before (white bars) and after (black bars) stimulation with $\mathrm{G}-\mathrm{CSF}$ and the M1/M2 ratio in control individuals (d) and in patients with type 2 diabetes (e). ${ }^{*} p<0.05$ vs controls in (d); $\dagger p<0.05$ vs pre-GCSF levels

literature. In addition, this definition does not distinguish between M1 and M2 cells. We therefore analysed more definite monocyte polarisation phenotypes, using antigenic profiles derived from studies on the monocytemacrophage differentiation cascade [15] to identify circulating cells more closely resembling tissue macrophages. Cells co-expressing CD68 (an LDL- and lectin-binding scavenger protein) and CCR2 (MCP-1 receptor) were considered pro-inflammatory M1 cells, while cells co-expressing CX3CR1 (fractalkine receptor) and the scavenger receptors CD163 or CD206 were considered anti-inflammatory M2 [14]. Based on these antigenic profiles, M1 cells are expected to enter the diseased vascular wall following MCP-1 gradients and to become foam cells [17], while M2 cells should patrol the vasculature and clean up the tissue via scavenger activity [14]. We performed a series of experiments to validate these phenotypes: M2 cells, compared with M1, were fourfold enriched in non-classical monocytes involved in the resolution of inflammation, and showed a lower TNF- $\alpha$ content and higher capacity for cleaning acetylated LDL. Moreover, the circulating M2 phenotype resembled in vitro polarised M2 macrophages, which were $\mathrm{CX} 3 \mathrm{CR} 1^{+} \mathrm{CD} 163^{+} \mathrm{CD} 206^{+}$. Finally, M1 cells had a much stronger capacity for transendothelial migration than M2 cells, suggesting that they are more 
prone to enter the diseased vessel wall. Therefore, the selected M1 and M2 monocyte phenotypes truly reflect cells with different inflammatory potential and resemble the corresponding macrophage populations.

We found that type 2 diabetes is characterised by a marked reduction in M2 cells while M1 cells were unchanged compared with controls; as a result, the M1/M2 polarisation ratio was increased in diabetes. As type 2 diabetes is considered a pro-inflammatory condition, it is striking that the monocyte polarisation imbalance is attributable to a defect in anti-inflammatory cells, rather than an excess of pro-inflammatory ones. This observation is, however, in line with the theory that diabetes is a disease of impaired damage control [31], in which injury is worsened by defective repair. The M1/M2 polarisation ratio was directly correlated to waist circumference, an indicator of central obesity and insulin resistance, and to $\mathrm{HbA}_{1 \mathrm{c}}$, an indicator of glucose control. In addition, the pattern of complications showed trend associations with altered polarisation. The presence of microangiopathy (particularly nephropathy) was associated with a reduction in M2 and an increase in the $\mathrm{M} 1 / \mathrm{M} 2$ ratio. Despite previous studies indicating a prominent role for M1/M2 cells in atherosclerosis [14, 32], we found no association with diabetic macroangiopathy, but the relationships between M1/M2 and diabetic complications should be confirmed in a larger cohort. Experimental studies indicate that polarisation of kidney macrophages is related to renal injury and function [33]. Only two diabetic patients had macroalbuminuria and all those with reduced eGFR had mild renal failure, thus preventing any correlation with the severity of nephropathy. Nonetheless, this is the first report of an association between diabetic nephropathy and altered monocyte polarisation. The levels of intermediate $\mathrm{CD} 14^{++} \mathrm{CD} 16^{+}$cells, to which most M1 and M2 cells belong, were shown to be predictive of cardiovascular outcomes in patients with chronic kidney disease [34]. As M1 and M2 are more detailed phenotypes than traditional monocyte subsets, the prognostic meaning of the M1/M2 ratio warrants investigation. Interestingly, decrease in M2 and increase in the M1/M2 ratio was already evident in drug-naive patients with short disease duration.

Recent data indicate that diabetes induces BM microangiopathy, with features similar to those seen in the kidney and in the retina [35-37]. In turn, microangiopathy causes BM dysfunction and alters stem-cell regulation [23]. Thus, we analysed monocyte subsets in BM aspirates of individuals with and without type 2 diabetes. First, it was noteworthy that the M1/M2 balance was inverted in BM compared with $\mathrm{PB}$, suggesting that monocyte subsets play a role in $\mathrm{BM}$ function [38]. In diabetes, M2 cells in the BM were significantly reduced compared with controls, but to a lesser extent than in the PB. Such a BM/PB gradient indicated a relative excess of M2 in the diabetic BM. This was confirmed by showing that G-CSF preferentially mobilises M2 cells only in diabetic patients, restoring the M1/M2 balance toward normal levels. All participants in the BM substudy had heart disease, thus limiting the generalisability of the findings to the general diabetic population. Notwithstanding this limitation, it appears that type 2 diabetes is associated with a depletion of circulating M2 cells, which are stuck in the BM and can be mobilised by pharmacological G-CSF doses.

Reversal of the M1/M2 imbalance in diabetes with BM stimulation indicates that $\mathrm{BM}$ dysfunction plays a role in diabetes-related inflammation and represents a possible link between distant end-organ complications. Speculatively, $\mathrm{BM}$ microangiopathy, by altering the monocyte polarisation status, might contribute to the development or progression of microangiopathy in other organs (Fig. 4). As monocytemacrophages are involved in vascular disease and regulate the cross-talks with inflammatory pathways [14], the M1/M2 imbalance in diabetes can exert a negative impact on the development of diabetic complications.

Thus, M1/M2 cells may be considered metabolicinflammatory biomarkers of the overall risk in diabetic patients. Of particular interest is the modulation of M1/M2 polarisation by lifestyle and glucose-lowering medications, particularly those with distinctive anti-inflammatory properties, such as glitazones and dipeptidyl peptidase-4 inhibitors. The prognostic meaning of the reduced M2 level in diabetic patients also warrants further investigation.

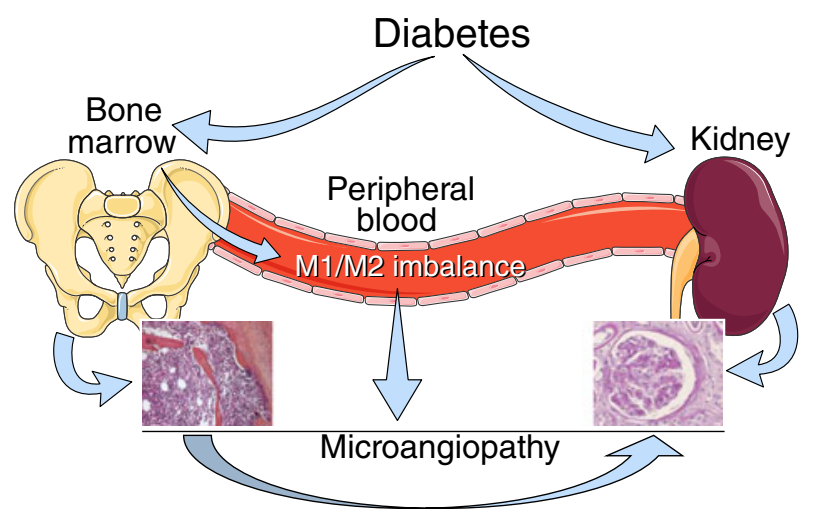

Fig. 4 Pathophysiological model. A model of the crosstalk between microangiopathy in the $\mathrm{BM}$ and in other organs through an imbalance in M1/M2 polarisation is shown. Diabetes induces pathological alterations in the BM similar to the microangiopathy observed in other organs such as the kidney. BM alterations seem to account for the altered M1/M2 polarisation status in PB. As M1/M2 imbalance is related to microangiopathy, especially nephropathy, it is tempting to speculate that BM and kidney microangiopathy are pathophysiologically related through dysregulation of BM-derived cells such as M1 and M2 monocytes 
Funding G. P. Fadini is supported by a European Foundation for the Study of Diabetes (EFSD)/Sanofi Clinical Investigator Fellowship.

Duality of interest The authors declare that there is no duality of interest associated with this manuscript.

Contribution statement GPF, SVdK, AC, CA and AA were involved in the conception and design of this study. Acquisition of data was carried out by GPF, SVdK, EB, MA, RC, NK, UL, AT, CB, FS, $\mathrm{SD}$ and $\mathrm{AZ}$. Analysis and interpretation of data was carried out by GPF, SVdK, EB, MA, RC, NK and UL. The article was drafted by GPF, SVdK and AA and was revised by EB, MA, RC, NK, UL, AT, $\mathrm{CB}, \mathrm{AC}, \mathrm{FS}, \mathrm{SD}, \mathrm{AZ}$ and $\mathrm{CA}$. All authors provided final approval of the version to be published.

\section{References}

1. Wellen KE, Hotamisligil GS (2005) Inflammation, stress, and diabetes. J Clin Invest 115:1111-1119

2. Neels JG, Olefsky JM (2006) Inflamed fat: what starts the fire? J Clin Invest 116:33-35

3. Shoelson SE, Lee J, Goldfine AB (2006) Inflammation and insulin resistance. J Clin Invest 116:1793-1801

4. Ceolotto G, Gallo A, Sartori M et al (2001) Hyperglycemia acutely increases monocyte extracellular signal-regulated kinase activity in vivo in humans. J Clin Endocrinol Metab 86:1301-1305

5. Ceolotto G, Gallo A, Miola M et al (1999) Protein kinase C activity is acutely regulated by plasma glucose concentration in human monocytes in vivo. Diabetes 48:1316-1322

6. Ceolotto G, Bevilacqua M, Papparella I et al (2004) Insulin generates free radicals by an $\mathrm{NAD}(\mathrm{P}) \mathrm{H}$, phosphatidylinositol 3'kinase-dependent mechanism in human skin fibroblasts ex vivo. Diabetes 53:1344-1351

7. Fernandez-Real JM, Ricart W (2003) Insulin resistance and chronic cardiovascular inflammatory syndrome. Endocr Rev 24:278-301

8. Libby P (2002) Inflammation in atherosclerosis. Nature 420:868874

9. Navarro-Gonzalez JF, Mora-Fernandez C (2008) The role of inflammatory cytokines in diabetic nephropathy. J Am Soc Nephrol $19: 433-442$

10. Kern TS (2007) Contributions of inflammatory processes to the development of the early stages of diabetic retinopathy. Exp Diabetes Res 2007:95103

11. Vincent AM, Callaghan BC, Smith AL, Feldman EL (2011) Diabetic neuropathy: cellular mechanisms as therapeutic targets. Nat Rev Neurol 7:573-583

12. Sica A, Mantovani A (2012) Macrophage plasticity and polarization: in vivo veritas. J Clin Invest 122:787-795

13. Mantovani A, Sica A, Sozzani S et al (2004) The chemokine system in diverse forms of macrophage activation and polarization. Trends Immunol 25:677-686

14. Mantovani A, Garlanda C, Locati M (2009) Macrophage diversity and polarization in atherosclerosis: a question of balance. Arterioscler Thromb Vasc Biol 29:1419-1423

15. Charo IF (2007) Macrophage polarization and insulin resistance: PPARgamma in control. Cell Metab 6:96-98

16. Aron-Wisnewsky J, Tordjman J, Poitou C et al (2009) Human adipose tissue macrophages: $\mathrm{m} 1$ and $\mathrm{m} 2$ cell surface markers in subcutaneous and omental depots and after weight loss. J Clin Endocrinol Metab 94:4619-4623
17. Chinetti-Gbaguidi G, Baron M, Bouhlel MA et al (2011) Human atherosclerotic plaque alternative macrophages display low cholesterol handling but high phagocytosis because of distinct activities of the PPARgamma and LXRalpha pathways. Circ Res 108:985995

18. Ziegler-Heitbrock L, Ancuta P, Crowe S et al (2010) Nomenclature of monocytes and dendritic cells in blood. Blood 116:e74-e80

19. Wong KL, Tai JJ, Wong WC et al (2011) Gene expression profiling reveals the defining features of the classical, intermediate, and nonclassical human monocyte subsets. Blood 118:e16-e31

20. Bories G, Caiazzo R, Derudas B et al (2012) Impaired alternative macrophage differentiation of peripheral blood mononuclear cells from obese subjects. Diab Vasc Dis Res 9:189195

21. Satoh N, Shimatsu A, Himeno A et al (2010) Unbalanced M1/M2 phenotype of peripheral blood monocytes in obese diabetic patients: effect of pioglitazone. Diabetes Care 33:e7

22. Expert Committee on the Diagnosis and Classification of Diabetes Mellitus (2003) Report of the expert committee on the diagnosis and classification of diabetes mellitus. Diabetes Care 26(suppl 1):S5-S20

23. Fadini GP, Albiero M, Vigili de Kreutzenberg S et al (2012) Diabetes impairs stem cell and proangiogenic cell mobilization in humans. Diabetes Care 35:1-8

24. Krankel N, Kuschnerus K, Madeddu P, Luscher TF, Landmesser U (2011) A novel flow cytometry-based assay to study leukocyte-endothelial cell interactions in vitro. Cytometry A $79: 256-262$

25. Martinez FO, Gordon S, Locati M, Mantovani A (2006) Transcriptional profiling of the human monocyte-to-macrophage differentiation and polarization: new molecules and patterns of gene expression. J Immunol 177:7303-7311

26. Weiner LM, Li W, Holmes M et al (1994) Phase I trial of recombinant macrophage colony-stimulating factor and recombinant gamma-interferon: toxicity, monocytosis, and clinical effects. Cancer Res 54:4084-4090

27. Thomas J, Liu F, Link DC (2002) Mechanisms of mobilization of hematopoietic progenitors with granulocyte colony-stimulating factor. Curr Opin Hematol 9:183-189

28. Poitou C, Dalmas E, Renovato M et al (2011) CD14dimCD16 and $\mathrm{CD} 14^{+} \mathrm{CD} 16^{+}$monocytes in obesity and during weight loss: relationships with fat mass and subclinical atherosclerosis. Arterioscler Thromb Vasc Biol 31:2322-2330

29. Yang M, Gan H, Shen Q et al (2012) Proinflammatory $\mathrm{CD} 14^{+} \mathrm{CD} 16^{+}$monocytes are associated with microinflammation in patients with type 2 diabetes mellitus and diabetic nephropathy uremia. Inflammation 35:388-396

30. Patino R, Ibarra J, Rodriguez A et al (2000) Circulating monocytes in patients with diabetes mellitus, arterial disease, and increased CD14 expression. Am J Cardiol 85:1288-1291

31. Schaper NC, Havekes B (2012) Diabetes: impaired damage control. Diabetologia 55:18-20

32. Bouhlel MA, Derudas B, Rigamonti E et al (2007) PPARgamma activation primes human monocytes into alternative M2 macrophages with anti-inflammatory properties. Cell Metab 6:137-143

33. Ma LJ, Corsa BA, Zhou J et al (2011) Angiotensin type 1 receptor modulates macrophage polarization and renal injury in obesity. Am J Physiol Renal Physiol 300:F1203-F1213

34. Rogacev KS, Seiler S, Zawada AM et al (2011) $\mathrm{CD} 14^{++} \mathrm{CD} 16^{+}$ monocytes and cardiovascular outcome in patients with chronic kidney disease. Eur Heart J 32:84-92 
35. Spinetti G, Cordella D, Fortunato O et al (2013) Global remodeling of the vascular stem cell niche in bone marrow of diabetic patients: implication of the miR-155/FOXO3a signaling pathway. Circ Res 112:510-522

36. Oikawa A, Siragusa M, Quaini F et al (2010) Diabetes mellitus induces bone marrow microangiopathy. Arterioscler Thromb Vasc Biol 30:498-508
37. Fadini GP (2011) Is bone marrow another target of diabetic complications? Eur J Clin Invest 41:457-463

38. Chow A, Lucas D, Hidalgo A et al (2011) Bone marrow CD169 macrophages promote the retention of hematopoietic stem and progenitor cells in the mesenchymal stem cell niche. J Exp Med 208:261-271 